\title{
Fairness as "Appropriate Impartiality" and the Problem of the Self-Serving Bias
}

\author{
Charlotte A. Newey ${ }^{1}$ (D)
}

Accepted: 17 November 2015 / Published online: 12 December 2015

(C) The Author(s) 2015. This article is published with open access at Springerlink.com

\begin{abstract}
Garrett Cullity contends that fairness is appropriate impartiality (See Cullity (2004) Chapters 8 and 10 and Cullity (2008)). Cullity deploys his account of fairness as a means of limiting the extreme moral demand to make sacrifices in order to aid others that was posed by Peter Singer in his seminal article 'Famine, Affluence and Morality'. My paper is founded upon the combination of (1) the observation that the idea that fairness consists in appropriate impartiality is very vague and (2) the fact that psychological studies show the self-serving bias is especially likely to infect one's judgements when the ideas involved are vague. I argue that Cullity's solution to extreme moral demandingness is threatened by these findings. I then comment on whether some other theories of fairness are vulnerable to the same objection.
\end{abstract}

Keywords MoralDemandingness · Fairness $\cdot$ Impartiality $\cdot$ Moral Obligation · Self-Serving Bias - Cullity, Broome $\cdot$ Murphy $\cdot$ Poverty Alleviation

\section{Introduction}

Garrett Cullity claims that all unfair actions share a common feature (Cullity 2008): 'Unfair actions are failures of appropriate impartiality; fair actions are those that are not unfair.' Cullity applies his account of fairness to derive a limit on the amount of resources the affluent are morally required to devote to alleviating global poverty (Cullity 2004). In this paper, I do not seek to demonstrate that Cullity's account of fairness is wrong in general. However, I do take issue with the practical application of Cullity's account of fairness to limit Singer's extreme moral demand. And I think that the problems arising here spread.

I start by setting out the key features of Cullity's account of fairness as appropriate impartiality. The inherent vagueness in this account is noted. I then identify four aspects of the self-serving bias that are relevant to practical considerations of fairness. I argue that these

Charlotte A. Newey

neweyc@cardiff.ac.uk

1 Philosophy Department, Cardiff University, John Percival Building, Colum Drive, Cardiff CF10 3AT, UK 
four aspects of the self-serving bias are particularly relevant to the practical application of Cullity's account of fairness. Cullity uses his account of fairness to limit the extreme moral demand posed by Peter Singer (Singer 1972) but I demonstrate that both Cullity's own fairness-based limitation of demandingness and his advice to agents are open to distortion by self-serving biases. Cullity's account provides too much opportunity for self-serving biases to distort people's (sincere) conclusions about what fairness requires. So the practical application of Cullity's account of fairness can spark conflict rather than resolve it. But we appeal to fairness to be effective in resolving conflict rather than provoking or intensifying it. I close the paper with a sketch of how the problem of self-serving biases might generalise beyond Cullity's account of fairness.

\section{Fairness and Appropriate Impartiality}

How could one argue for the thesis that unfair actions are failures of appropriate impartiality and fair actions are those that are not unfair? ${ }^{1}$ Cullity points to the correlation between unfairness and failures of one or another kind of impartiality. And there is no denying that a familiar pre-theoretical view is that fairness is tightly connected with impartiality.

Cullity recognizes that there are indefinitely many different ways of being impartial (Cullity 2008). We cannot plausibly hold that just any impartial way of making a decision would be a fair way of making that decision. For example, rolling a die is undeniably an impartial way to allocate grades to students' essays, but it is not a fair way to allocate grades. Fairly allocating grades to essays requires more than simple impartiality; it requires the marker to be insensitive to certain qualities of the students, such as accent, gender, social class, etc., but sensitive to differences in the qualities of the papers being graded. Because fairly allocating grades to essays requires sensitivity to differences in the qualities of the papers being graded, rolling the die bypasses the kinds of evaluation that are appropriate for allocating grades.

What makes a form of impartiality appropriate or (as in the example of rolling die in order to allocate grades to students' essays) inappropriate? Cullity offers no general method by which to determine the type of impartiality that would be appropriate. He claims instead that what determines the appropriate kind of impartiality is an independent question answerable only within a more general normative theory (Cullity 2008). However, he points out that different distributive practices are governed by their different aims. He suggests that, at least often, the aim of the practice determines which kind of impartiality is appropriate (Cullity 2008).

\section{Easy Cases, Complex Cases and Vagueness}

Sometimes, it is easy to identify the aim of the practice. In a $100 \mathrm{~m}$ race, the aim is to give people in a certain group the opportunity to prove that they can sprint faster over $100 \mathrm{~m}$ than others in their group. Where identifying the aim of the practice is easy, identifying the appropriate form of impartiality is usually straightforward. If the race is the 'Boys Under-13

\footnotetext{
${ }^{1}$ I set aside the fact that many actions are neither fair nor unfair. I also note that partiality and impartiality are contraries, not contradictories. A failure to act impartially does not necessarily mean that one acts partially; partiality is irrelevant to certain actions. For example, when I clean my teeth or read a newspaper I am not acting unfairly but the lack of unfairness does not mean that I am acting fairly. Fairness and partiality are irrelevant to these actions.
} 
$100 \mathrm{~m}$ sprint', race officials are not flouting the requirements of impartiality when they exclude all females and anyone aged over 13. Once the qualification criteria are met and race conditions and rules applied equally to all entrants, there is only one criterion for the officials to apply, namely the order in which the contestants cross the finishing line. Being appropriately impartial in officiating the race requires being uninfluenced by facts such as who put in the most effort or who would benefit most from a win or who has the nicest smile.

In sports-related, rule-governed examples, it is often clear what kind of impartiality is required and, in contrast, what facts are irrelevant. And in practices where the appropriate form of impartiality is easy to identify, it is likely to be easy to identify what would make judgments fair or unfair. ${ }^{2}$ However, in more complex cases, we need to know whether impartiality is a requirement at all and, if so, what kind of impartiality. If we misidentify the appropriate kind of impartiality, then we will presumably reach the wrong conclusions about what fairness requires. More complex cases increase the likelihood of misidentifying the appropriate kind of impartiality by introducing competing factors, which may or may not be relevant to reaching a judgement about what fairness requires. If we are to apply Cullity's account of fairness correctly, we therefore need to provide sufficient guidance for agents to focus only on relevant factors and to give those factors the correct weight.

Disputes about fairness typically arise in cases that involve some complexity and competing considerations so let us move away from simple game structures to more complicated judgements about fairness. Opportunities to misidentify what fairness requires arise both in terms of what form of impartiality ought to be applied and, since impartiality is scalar, what degree of impartiality ought to be applied. However, only one form of impartiality will be appropriate, according to Cullity (2008: 3): 'Judgements about fairness and unfairness, I claim, concern actions for which one particular way of being impartial is morally required. ${ }^{3}$

Complex cases provide two challenges for Cullity's account. The first challenge is that of identifying what form of impartiality will be appropriate in a dispute concerning fairness. In the remainder of this section, I concentrate on this first challenge. I will then focus on the challenge raised by the self-serving bias. This bias is a tendency to conflate what is fair with what benefits oneself (Babcock and Loewenstein 1997). ${ }^{4}$

Suppose Daniel is a keen amateur photographer and his good friends know that he is trying to break into professional photography in order to supplement his low income. Daniel's good friend Chloe asks him to photograph her wedding and Daniel agrees. Unfortunately Daniel is inexperienced in business. As a result, Daniel and Chloe do not agree any details prior to Chloe's wedding, including Chloe's expectations for the day or a fair price for the job. Chloe simply says that she will pay him to take 'candid shots' throughout the day. At the wedding, Daniel takes over five hundred photographs, which he spends many hours editing before he shows Chloe the pictures, leaving her with an invoice for $£ 400$, less than one-third of the price that local professional photographers would charge. Chloe refuses to pay $£ 400$, claiming that the photographs were far more elaborate and plentiful than she wanted and that she thought a friend would make sure not to run up the cost.

Let us set to one side the fact that prudentially there are many things that Daniel and Chloe should have done. This kind of dispute about fairness, where friends have different expectations of each other and are reluctant to start out on a professional basis, is very common. This

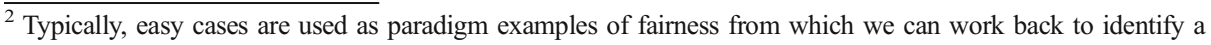
common feature or a requirement of fairness. See Cullity (2004), (2008) and Broome (1990/91), (1998).

${ }^{3}$ My emphasis.

${ }^{4}$ Hereafter, B\&L.
} 
is also an example to which there is no obvious answer. We cannot simply say that this is a legal issue, since the two friends did not make an unambiguous contract. We cannot resort to whatever the market rate would be. In this type of case, there is not an obvious market rate for a part-time photographer who has skill but no portfolio, and there is no universally acceptable discount rate for friends and family.

For the sake of argument, let us agree that the quality of photographs was good, even if the number and style was a point of disagreement. The following factors are relevant to fairness. First, there was an informal agreement that Daniel would do a job for Chloe and that Chloe would give him some money in return for it. Despite the absence of a formal contract, some degree of reciprocity exists between Daniel and Chloe. Next, consider the obligations of friendship. Daniel thought that Chloe was helping a friend out by employing him; he thought that he was helping her out by giving her so many photographs at a rate she would not be able to get elsewhere. Chloe thought that she was helping Daniel by giving him experience and an opportunity to develop a portfolio, but she also expected that, as a friend, he would charge only a nominal sum. Finally there is a question of desert. Daniel put much time and effort into taking and editing the photographs and the quality was good. Chloe's reaction was that he put more time and effort into taking the photographs than she expected.

If faimess is appropriate impartiality then, in order to establish a fair solution to Daniel and Chloe's predicament, we need to determine what form of impartiality is appropriate in this case. A good starting point must be that each ignores their personal desires about the price of the photographs. That Daniel wants to be paid $£ 400$ for the photographs and that Chloe wants them for a nominal sum are irrelevant to the question of what would be a fair price. So we turn to the requirements of reciprocity, friendship and desert. Even if Daniel and Chloe agree that reciprocity, friendship and desert should be part of determining a fair price for the photographs, they may yet have different ideas about what reciprocity, friendship and desert require, and about which of these considerations, if any, is the most important.

If Chloe's view of the requirements of reciprocity is that one good turn deserves another, then she might not only give Daniel a small amount of money but also later do some favour for Daniel. In contrast, if Daniel thinks reciprocity requires immediate reciprocal action, then he expects Chloe to pay a higher amount for the service now. ${ }^{5}$ So Chloe and Daniel take different views about what reciprocity requires. As I will demonstrate in section 4, if Daniel and Chloe are under the influence of the self-serving bias, they will both think that they are judging the matter fairly and impartially. If we did not know that each was influenced by the self-serving bias, we might allow that both Daniel and Chloe were indeed judging the matter in an appropriately impartial way.

If Daniel and Chloe are going to apply an appropriate form of impartiality to the degree of influence that friendship should have, they need to abstract their judgment from their own friendship and ask what friendship in general requires. But is that really fair? Does not the strength of friendship vary from case to case? Might fairness require one judgment in a dispute between lifelong friends and another between recently acquainted but enthusiastic friends? Perhaps Daniel and Chloe should ask what other, similar, friendships would require. Perhaps both could agree that friendship in general would require that both friends should make a degree of sacrifice. Often when friends have work done at 'mates' rates', the labourer sacrifices some income and the person who is paying gets a lower price than the 'arms-length' rate..

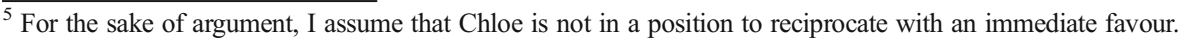


Finally, considering desert, Daniel and Chloe might agree that both productivity and effort should be rewarded. In the actual case they are unlikely to agree about the degree to which productivity and effort should be rewarded. Perhaps, if they thought about their situation impartially, they could see that Chloe was disingenuous when she said she just wanted twenty snaps and that Daniel was over enthusiastic to take five hundred images. Maybe some research would show that, typically, wedding photographers provide $n$ images of a wedding. However, we will see that research suggests that neither Daniel nor Chloe is likely to identify a number of photographs that sounds fair or impartial to the other, even though both believe themselves to be sincere in their attempts to do so.

This example of Daniel and Chloe demonstrates that there are various forms that appropriate impartiality might take. Perhaps the appropriate form of impartiality is to reward desert to a certain degree or to fulfil the requirements of reciprocity in a particular way. Perhaps the appropriate form of impartiality is compliance with the requirements of friendship. Or it might be appropriate to give some weight to the importance of friendship but to give similar weight to matters of desert and none to reciprocity. But, obviously, the manner in which Daniel and Chloe are left to establish what might be appropriate is fraught with difficulties. Moreover, it is exactly cases like these that the self-serving bias is most likely to intrude, and in ways not yet obvious to the parties involved.

Recall Cullity's advice that the aim of the practice will determine the form of impartiality that is appropriate to apply. In the example of Daniel and Chloe, there is no clearly identifiable relevant practice, certainly not one with an aim. Admittedly, there is a practice of hiring professional photographers with whom one has an arms-length relationship, and there is a practice of getting married without any professional photographers but with some friends who are likely to take photographs. But there is no 'practice' of hiring friends who aspire to be professional photographers. A significant number of disputes about fairness are like this example in that appropriate impartiality is not dictated by the aim of a relevant social practice. Instead, multiple considerations are relevant to fairness, and these considerations come in different degrees of importance.

One might think that this complication is limited to informal disputes and that the issue of fairness will be more clear-cut when we consider established practices. Yet established practices very often contain a number of competing considerations, with no method of identifying the weight and relevance of each consideration. So the problem I have focused on has very wide scope.

Turn now to a different example. Oxfam clearly states three aims: 'We aim to save lives by responding quickly with aid and protection during emergencies, empower people to work their own way out of poverty, and campaign for lasting change. ${ }^{6}$ Given that Oxfam of course has only limited resources, which one of Oxfam's aims dictates the form of impartiality appropriate to determining a fair distribution of aid resources? When is it fair to direct resources to campaigning for long-term changes that will benefit future generations at the expense of the suffering of existing generations? If Cullity's account is to generate a clear indication of what fairness requires of Oxfam, Oxfam must have either a single overarching aim or a strict ranking of the three aims identified.

To keep matters simple, let us suppose Oxfam's overarching aim is to satisfy the needs of the worst off. How do we get from this aim to the form of impartiality that is appropriate? First, the definition of 'need' is itself highly contestable. ${ }^{7}$ Second, kinds of suffering vary dramatically, and there is persistent uncertainty about who is worst off because of uncertainty about what constitutes one person's being better or worse off than someone else. Third, prioritising the prevention of

\footnotetext{
${ }^{6}$ http://www.oxfam.org.uk/what-we-do/about-us/our-vision-values-and-goals/our-aims-and-approach

${ }^{7}$ See Wiggins (1998) Braybrooke (1987); Miller (1999); Thomson (2005).
} 
poverty in the future over disaster relief could save more lives in the long run, but there is of course a controversy about how much, if at all, Oxfam should discount the needs of future people, given the uncertainties about technological change, global warming, etc. Because of these three reasons, even if the aim of a practice can identify the appropriate form of impartiality to use and even if Oxfam's aim is to satisfy the needs of the worst off, it is very unclear which form of impartiality is appropriate for Oxfam's use. If we cannot identify that form of impartiality, then we cannot apply Cullity's theory of fairness. Simply identifying the aim of Oxfam's practice does not offer the kind of guidance necessary for Oxfam to distribute their resources fairly.

Cullity's account of fairness makes space for a wide range of interpretations about what might be fair. While some philosophers see this interpretive space as an essential element to any theory that is intended to cover a wide variety of cases, others would object that such vagueness and lack of clear guidance renders Cullity's account untenable. I wish to stress that I am not setting out to reject Cullity's general account of fairness. A good theory of fairness may have to leave room for judgement and even a very good theory of fairness may be difficult to apply in many cases. Rather, I contend that the vagueness and resulting lack of clear guidance threaten the successful practical application upon which Cullity's limitation of moral demandingness depends. I will now demonstrate how cognitive biases provide a second and powerful challenge to the successful application of Cullity's account of fairness.

\section{The Self-Serving Bias}

A psychological mechanism that has received attention from experimental psychologists and economists is the self-serving bias in judgements about fairness. Recently economists have focused on the self-serving bias as a cause of negotiation impasses (Babcock and Loewenstein 1997) and as a factor in disagreements about the solution to climate change (Kriss et al. 2011).

The specific area of research into the self-serving bias that is important for my purposes relates to how people make systematic errors when making judgements about fairness, i.e., when people sincerely think that what is beneficial to themselves is also fair to all parties concerned. I have identified four findings from the psychological research into the self-serving bias that are particularly relevant to philosophical investigations of fairness. While I explore the wider implications of these findings in section 5, I here identify aspects of the research into self-serving biases that connect closely to Cullity's account of fairness.

\subsection{The Bias is Subconscious}

When evaluating alternative possible actions, people tend subconsciously to identify the action that would probably be most beneficial to themselves. Next they notice that some considerations relevant to fairness count in favour of that action. Then they subconsciously emphasize the relative importance of those considerations and consciously appeal to those considerations in attempting to justify preferring that action (Shu and Bazerman 2012).

\subsection{People Identify What They Think of as Fair Actions as Impartially Justified, Even if Their Endorsing These Action is Self-Serving}

Despite the biased process identified in (4.1), people are sincere in their judgments. For example, they believe that they are endorsing a genuinely fair action and they also believe 
that 'their personal conception of fairness is impartial' (Babcock and Loewenstein 1997). This is a particular problem for the practical application of Cullity's account. Agents who think about fairness in terms of impartiality remain equally vulnerable to self-serving cognitive impediments to truly fair judgements.

\subsection{In Simple Cases, the Self-Serving Bias is not Usually Present}

If two agents are asked to distribute a good fairly between them, then absent any further information they will both split the good equally. Using simple cases such as games, races, allocation of prizes, and turn taking, Cullity correctly identifies the connection between fairness and impartiality. Of course, people tend to reach consensus about fairness in such cases. These cases are nonetheless informative and give support to Cullity's claim that fairness is essentially connected to some kind of impartiality.

\subsection{The Self-Serving Bias Becomes More Evident as Situations Become More Complex ${ }^{8}$}

Factors that increase complexity could be additional information about the situation or further criteria to be applied. For example, the subjects might be told to divide a sum of money fairly between themselves and a much richer person or between themselves and a person who is in much greater need as a result of having been reckless. The additional information about the second person gives the subjects an opportunity to be influenced by it or to ignore it, introducing new criteria for their judgment of fairness. Perhaps they should take into account the other person's need or irresponsible attitude. Now, subjects who think that a fair distribution of the sum requires taking need or desert into account may be employing an appropriate form of impartiality; on the other hand, they may simply be influenced by the self-serving bias.

Consider a case in which the appropriate form of impartiality is less sensitive to desert than need. A fair division of a sum of money would give more to second person, acknowledging their need and discounting their recklessness. However, with the additional criteria available, the self-serving bias can induce an agent to focus instead on desert, to a greater or lesser degree. Focusing on desert will reduce the sum to be paid to the second person, benefitting the agent who is making the distribution. If additional criteria are available, there will be greater opportunities for the self-serving bias to intrude. Without guidance as to which criteria are important and to what degree they are important, an agent could misidentify irrelevant factors - age, gender, membership of one's social group - as relevant to the decision. ${ }^{9}$

The problem that I am focusing on appears once there are disputes about what fairness requires. As disputes escalate, becoming more intractable, there is likely to be far more complexity. This complexity can be generated by vagueness about which criteria are relevant or about how to identify and interpret relevant criteria. Protracted climate change negotiations

\footnotetext{
${ }^{8}$ In the psychological literature, the kinds of situations I call 'complex' are called 'asymmetrical'. However, they are not asymmetrical in the sense that one party has more information or more options than another. Rather they are cases in which there is a range of possible solutions, some of which favour one agent; some of which favour the other. The range of solutions generated is greater in complex cases such as the examples involving Daniel and Chloe and Oxfam. For example, one agent focuses on desert, but another focuses on reciprocity. Where there is a greater range of solutions, the self-serving bias is likely to have a more damaging effect.

${ }_{9}$ Perhaps age, gender and membership of a social group are the most relevant factors. But if the self-serving bias is in operation, an agent is influenced by the wrong factors and for the wrong reasons.
} 
are hardly surprising if we consider the range of different policy proposals that are animated by different views of what fairness requires. Some advocate on equal limits on $\mathrm{CO}_{2}$ emissions per capita; others advocate gearing limits on $\mathrm{CO}_{2}$ emissions to historic emissions levels.

We should not give up on the project of applying an account of fairness. However, practical application of an account of fairness needs to be insulated from the effects of the self-serving bias. Since the bias is more likely to be present in complex cases, an account of fairness suitable for practical application should find ways to reduce complexity. To return to a recent example, dividing money fairly between a rich person and a poor but reckless person has a layer of complexity that could be reduced by instructing the agent or agents to focus on need or desert. Thus, if Cullity's account offered clear guidance about the forms and degrees of impartiality that would be appropriate in various circumstances, the self-serving bias would have less opportunity to affect judgements and cause conflict. But Cullity does not set out to give such guidance (Cullity 2008):

Although I have offered a simple and general unitary description of unfair action, I offer no simple and general unitary explanation of when and why different forms of impartiality are appropriate.

Cullity's advice is restricted to identifying the appropriate forms of impartiality by considering the aim of the relevant social practice. But some social practices do not have an aim at all, while the Oxfam example demonstrates that other social practices have multiple aims with no clear priority ranking. Moreover, the aims that some social practices have might require considerable interpretation. In all of these cases, the self-serving bias can worm its way in and influence the selection of an inappropriate form of impartiality, and thus adversely influence judgement about what is fair.

These four features of the self-serving bias ((i) being present subconsciously, (ii) identifying the self-serving solution as impartial, (iii) being absent from simple cases and (iv) being prevalent in complex cases) are important determinants in the likelihood of the self-serving bias affecting judgments of fairness. Armed with this knowledge, we can understand why Daniel and Chloe failed to reach consensus about a fair solution to the wedding photography problem. We have already seen the complex nature of the case and the lack of guidance available to help Daniel and Chloe identify an appropriate form of impartiality. If either or both were under the influence of subconscious self-serving biases, each would sincerely identify their own self-serving solution as impartial and fair and view the other's solution as biased and unfair.

\section{The Fairness Solution to Moral Demandingness}

I now turn to Cullity's proposed solution to the problem of moral demandingness concerning global poverty, starting with a summary of the argument Cullity puts forward in The Moral Demands of Affluence (Cullity 2004).

Cullity uses a two-stage strategy to attempt to replace Singer's extreme demand with a less demanding principle. The first stage is an argument from beneficence that morality cannot require us to live a purely altruistically focused life. The second stage is an argument that contributions towards global poverty are limited by the requirements of fairness. My focus is on the second stage and I maintain that both the second stage of the argument and Cullity's subsequent advice to individual agents could be affected by the self-serving bias.

We have seen that complex judgements about fairness are more likely to be affected by the self-serving bias and also that Cullity's procedure for making judgements about fairness 
provides little guidance in complex cases. When Cullity himself uses fairness to limit Singer's extreme moral demand, we cannot be certain that his method is unaffected by the self-serving bias. ${ }^{10}$ The opportunity for the self-serving bias to affect Cullity's limitation of moral demandingness is more easily revealed if we consider the argument in more depth:

Premise 1: If anyone should keep donating to aid agencies until the point where making a further contribution would require of him or her a moral sacrifice comparable to the suffering he or she is trying to alleviate, it is immoral for anyone to live a nonaltruistically focused life.

Premise 2: Whenever I keep donating to aid agencies until the point where making a further contribution would require of me a moral sacrifice comparable to the suffering I am trying to alleviate, I am (almost always) helping someone else to live a nonaltruistically focused life.

Conclusion 1: Whenever I keep donating to aid agencies until the point where making a further contribution would require of me a moral sacrifice comparable to the suffering I am trying to alleviate, I am (almost always) helping someone to live a life it is immoral for her to live.

Premise 3: Morality cannot require me to help any agent to do something or live in a way that it is immoral for her.

Conclusion 2: Morality cannot require to keep donating to aid agencies until the point where making a further contribution would require of me a moral sacrifice comparable to the suffering I am trying to alleviate.

Supporting premise 2 of Cullity's argument is the assumption that non-altruistically focused lives are the main source of value for most of us. ${ }^{11}$ This assumption guides Cullity in his choice of what kind of impartiality is appropriate in determining our moral obligation to donate to aid programmes. Why is this the case? Cullity assumes that our reason for saving a life is grounded in certain non-altruistic interests of the agent whose life is at risk. Later, I will offer some of Cullity's examples of the goods that might make up such non-altruistically focused lives. For now, however, it suffices to say that these goods are life enhancing. In the next stage of his argument, Cullity turns to fairness as appropriate impartiality in order to determine what kinds or degrees of non-altruistically focused lives are morally permissible. He notes that lifeenhancing goods 'essentially involve personal partiality'. ${ }^{12}$ His suggestion is that any agent, whether affluent or poor, can only benefit if she nurtures her friendships and family relationships, participates in her culture, and pursues those worthwhile projects that have special connections to her.

Despite this personal partiality, provided every agent is permitted to pursue such goods, then a form of impartiality obtains. If every agent is permitted to pursue such goods, then personal features of agents such as their religion, age, location, race or gender do not restrict the pursuit of these goods. Thus every agent is impartially permitted to pursue partial, life-

\footnotetext{
${ }^{10}$ I do not claim that Cullity's conclusion about the moral demands of affluence is affected by the bias, only that it is possible. Moreover, if it were, Cullity and other members of 'the affluent' cannot be expected to recognize the influence of the self-serving bias, because of the manner in which it operates.

${ }^{11}$ See Cullity (2004) Chapter 7. In grounding the reason to save lives in other agents' non-altruistic pursuits, Cullity's position differs from Singer's, in which the reason for saving a life is that pain and unavoidable death are usually very bad and we ought to prevent such things when we can do so at comparatively little cost.

${ }^{12}$ My emphasis.
} 
enhancing goods. ${ }^{13}$ Cullity refers to this as complete impartiality (Cullity 2004) and concludes that fairness (understood as appropriate impartiality) dictates that neither the poor nor the affluent are acting immorally in pursuing non-altruistically focused lives. Agents are acting immorally, according to Cullity, if they fail to contribute to poverty alleviation when the resources they could contribute are not needed for the pursuit of their own life-enhancing projects. Cullity's complete impartiality is the form of impartiality that he believes to be appropriate in the case of global poverty but I dispute this by rejecting the presumption that complete impartiality is always, or in this case, superior to some other form of impartiality.

In Cullity's proposed solution to extreme demandingness, complete impartiality consists in applying the same principle to everyone, rich and poor alike, making no distinction between people because of their religion, race, gender or location. Note, however, that this form of impartiality is compatible with a number of principles which, in themselves, may exclude some people or favour others. ${ }^{14}$ Therefore we must specify Cullity's principle more clearly. His proposal is that a certain level of personal spending is permissible for all agents, rich or poor: the level of spending necessary to sustain each agent's own life-enhancing, non-altruistic projects.

However, there is no clear hierarchical value to degrees of impartiality. Should 'complete impartiality' always be the most superior form of impartiality? A simple example demonstrates that it should not. Suppose that, ignorant of what kind of impartiality was appropriate to grading students' papers, I opt to employ complete impartiality. I place all the papers in a box and, drawing blind, allocate grades at random. This kind of impartiality is greater (more impartial) than one in which I abstract from students' identities yet focus on the quality of their papers, but it is clearly inferior in this context. The spectrum of impartiality Cullity considers ranges from complete egoism, at the least impartial end, to a utilitarian form of impartiality that gives equal weight to each person's interests. Cullity specifies that the appropriately impartial point of view is (2004: 121), 'a point of view of... completely impartial concern for each person's interests'.

I reject the assumption that the appropriately impartial point of view from which to assess standards of beneficence is one of completely impartial concern for each person's, or indeed other peoples', interests. If global poverty alleviation is our concern, then is more plausible that the appropriate point of view in the global poverty debate would be a point of view that gives additional weight to the interests of the worse off for the very reason that it is the severity of their plight that grounds our concern. Furthermore, Cullity's focus on complete impartiality omits potentially relevant considerations for fairness. The focus on the permissibility of the pursuit of partial projects obscures the fact that, for anyone living in abject poverty, the possibility of pursuing such projects is non-existent. Moreover, allowing everyone, rich or poor, to limit contributions to life-saving projects by a sum that enables them to follow life-

\footnotetext{
${ }^{13}$ Calling such partiality a form of appropriate impartiality could sound paradoxical to some ears. One might think that partiality and impartiality are contraries. Cullity's understanding of impartiality is sufficiently protean to allow him to concede that someone's attitude toward others can be an instance of appropriate partiality and an instance of appropriate impartiality. When parents favour their own children to some acceptable degree, they are being appropriately partial. Parents should be partial to their children, perhaps not wholly, but to a certain extent and if every parent is permitted this degree of partiality, Cullity might refer to this practice as a form of appropriate impartiality.

${ }^{14}$ An example may help. An institutional rule that permits all members of the public to visit an art gallery appears to be applying a form of complete impartiality. If that art gallery can only be accessed by steps, a proportion of society is excluded. Simply applying one principle or rule is insufficient to ensure that all members of society are not only permitted to visit but also able to enter the gallery.
} 
enhancing projects is a form of impartiality that favours the affluent over the poor. It is plausible that life-enhancing projects generate reasons of beneficence to help others but lifeenhancing projects do not generate reasons to help others at the expense of providing lifesaving resources.

Cullity proposes that we should periodically review how much we have spent on ourselves and how much we have given to poverty alleviation and adjust our future spending and aid contributions in the light of our findings. Part of this exercise is to ensure that we identify that portion of our spending that can be justified in terms of appropriate impartiality. Cullity claims that the appropriate form of impartiality will be to limit the amount that we can spend on ourselves to whatever sum is needed to pursue our partial, life enhancing projects. This exercise is one of making a judgment according to the requirements of fairness. It requires that each agent can judge what is fair for her to retain to pursue her own life-enhancing goods, knowing that fairness permits both the affluent and the global poor to pursue partial lifeenhancing goods (Cullity 2004).

But this argument is too quick. Consider Cullity's discussion of the kinds of goods upon which the affluent may permissibly spend their resources. These goods include (Cullity 2004):

...the kind of expensive tertiary education that is available to relatively few people, globally speaking. For it is almost always reasonable to believe that this will substantially advance a person's understanding, achievements and participation in a culture, throughout her life.

Similarly, Cullity allows for expenditure on holiday travel, not justified by the pleasure that holidays typically bring, but because they are an opportunity to bond with family. He notes that expensive music lessons for the talentless might be unjustifiable in terms of the music they end up producing yet justifiable in terms of their participation in a culture: 'So expensive music lessons (or instruments) for the talentless seem hard to justify. However, up to a point, it seems sensible to see tuition in music, drama, and other arts as participation in a culture'.

The pursuit of tertiary education, holidays and means of participating in culture are valuable goods. However, can we be confident of the fair and impartial status of Cullity's conclusion that expenditure on these kinds of goods legitimately limits the contributions we ought to make to alleviate global poverty? Who is most likely to engage in the kind of lifeenhancing projects that Cullity describes, such as visiting museums, taking holidays and pursuing tertiary education? Even very modest kinds of life-enhancing activities are impossible for the 800 million people who suffered chronic undernourishment between 2012 and 2014. Thus I maintain that Cullity's proposed solution favours the affluent over the poor. Cullity's proposed solution ignores the reality that many inhabitants in developing countries do not have the means to feed themselves adequately, let alone pursue meaningful personal projects.

Suppose the life we can save is 'merely' one containing access to adequate nutrition, clean water, shelter, and protection from a range of basic threats such that the individual can work to provide for her family in future years. Suppose further that family bonding is restricted to the times spent preparing and eating the family meal and that participation in culture requires arduous work tending crops or digging wells. Suppose that education is basic and medical care is lacking. This life, devoid of holidays, rich cultural activities and tertiary education, is nonetheless a life worth saving. Cullity spends the first half of his (2004) The Moral Demands of Affluence defending the moral obligation to save the lives of the global poor. He agrees that such a modest life is a life worth saving. 
There are two opportunities for the self-serving bias to affect Cullity's method. First, I have noted that the self-serving bias might have influenced Cullity to apply the wrong kind of impartiality when assessing the acceptability of partial projects. To 1.6 billion people living in absolute poverty, a non-altruistically focused life in which basic needs such as food, shelter, medical care and dignity are met will be an enormous source of value. That their lives might be better still with more goods does not legitimise those goods for the affluent. The global poverty crisis is so severe that helping everyone currently living in absolute poverty to ascend above that state is unachievable with the current level of aid donations. Poverty is so severe that morality is extremely demanding, on Singer's view, even if the project is limited to providing the world's poorest people with basic subsistence goods. For Cullity's proposed application of fairness to succeed, the purpose of aid would need to be different. As the world is, Cullity's view permits a limit on life-saving resources, in order to pursue life-enhancing goods. That form of impartiality is not appropriate. A time may come when we do have a more ambitious aim for aid. When people in the world have access to clean water, food, shelter, education and certain legal protections, the function of aid might evolve to providing resources to help the worst off to pursue their own life enhancing partial projects. In that case, Cullity's appeal to the impartial moral status of the pursuit of partial goods would be legitimate and appropriate.

The self-serving bias has a second opportunity to distort judgements about what fairness requires when Cullity places each agent in charge of assessing what level of spending ought to be justifiable. Since the self-serving bias is so hard to detect, even sincere agents are likely to be mistaken in their choices. An appeal to fairness or impartiality to justify a holiday for the purposes of family bonding and the claim that expensive music lessons for the talentless may be justified because music enables participation in a culture may be judgments of fairness affected by the self-serving bias. Cullity is confident that we will be able to assess reasonably accurately the impact that different levels of spending will have on our lives but I do not share his optimism. Suppose I am considering whether to take my children to a summer school, to meet other people with similar interests, to bond with my family and to learn some new skills. I consider that I could stay home and donate to Oxfam the money I would have spent on the week away. I am allowed by Cullity's principle to spend this money on the summer school only if the time with my children will make a significant difference to our relationships, to our knowledge, or to our participation in our culture. The evidence concerning the incidence of the self-serving bias suggests that even if I sincerely try to make impartial judgements about the fairness of my spending level, I am likely to overestimate the difference that going on this trip will make to my children and me. Rather I will be influenced to choose in a self-interested manner.

\section{The Extent of the Problem}

I have focused on Cullity's account of fairness for two reasons. First, the literature on the selfserving bias reveals that the bias leads one to think not only that one's (self-serving) action is fair but also that it is impartially justified. Thus the way in which the bias operates is particularly salient for an account linking fairness and appropriate impartiality. Second, Cullity is one of the few authors who rely on the practical application of his account of fairness. While it is beyond the remit of a single paper to go into similar depth for all accounts of fairness, I consider briefly whether the problem of the self-serving bias is likely to plague other accounts of fairness in the same way and, indeed, whether the problem of the self-serving 
bias is likely to generalise beyond fairness. ${ }^{15}$ For simplicity, I will limit my discussion to the relation between fairness and global poverty.

Liam Murphy also relies on the practical application of fairness to limit moral demandingness. Murphy offers an account of what a fair share is and then claims that morality cannot require an agent to contribute more than her fair share to the alleviation of poverty. Murphy's 'Collective Principle of Beneficence' offers considerably more detailed guidance than Cullity's account of fairness as appropriate impartiality (2000):

Everyone is required to perform one of the actions that, of those available to her, is optimal in respect of expected aggregate weighted well-being, except in situations of partial compliance with this principle. In situations of partial compliance, a person's maximum level of required sacrifice is that which will reduce her expected level of wellbeing to the level it would be, all other aspects of her situations remaining the same, if there were to be full compliance from that point on. Under partial compliance a person is required to perform either an action, of those requiring no more than the maximum level of required sacrifice, that is optimal in respect of expected weighted aggregate wellbeing or any other action which is at least as good in respect of expected aggregated well-being.

Of course, calculating a fair share is likely to be complicated, meaning that there may be opportunities for the self-serving bias to operate in the assessment of what a fair share is and, perhaps, in assessing which course of action would maximise expected aggregate weighted well-being. Nonetheless, there is a sense in which this complexity may offer some protection. I cannot possibly calculate my share of the burden of global poverty alleviation might be, though I acknowledge that there will be such a thing. That job requires input and collaboration from multiple sources. ${ }^{16}$ If a solution can be found, then it may be (a) public and (b) taken out of the hands of the people whose interests would be served by favouring one calculation process over another. The publicity of the calculation may afford opportunities to reduce or at least to expose biases.

There is a second sense in which Murphy's fairness-based solution to poverty might be vulnerable to self-serving biases. Initially, Murphy views fairness as a pro tanto consideration but as he develops his solution, he affords fairness the weight of an all things considered moral judgement. As Michael Ridge writes (2011), 'While the moral principles advocated by Singer and Unger seem to ignore the arguably unfair burdens imposed on the conscientious moral agent, [Murphy's] collective principle seems to ignore the arguably unfair burdens imposed on the poor.' If the self-serving bias is effective in Murphy's proposed solution to moral demandingness, it may have obscured the point that not only fairness to potential contributors but also fairness to potential beneficiaries needs to be considered, a point that Ridge acknowledges.

John Broome's view (Broome 1990/91) that fairness requires that moral claims be satisfied in proportion to their strength depends, for successful application, on the accurate identification of moral claims and knowledge about the relative strength of claims. If disputes about fairness arise and we apply Broome's account of fairness, we might suppose that agents will be unable to agree what considerations count as claims or how strong those claims are. We could

\footnotetext{
${ }^{15} \mathrm{I}$ am indebted to two anonymous referees for pressing me on this issue.

${ }^{16}$ I set to one side the fact that a solution to global poverty is unlikely to be a straightforward monetary issue.
} 
rerun my example about Daniel and Chloe. Perhaps Daniel and Chloe fail to agree on what constitutes a claim or what the relative strengths of each other's claims are.

However, Broome's account at least focuses Daniel and Chloe's attention on the root of their disagreement. Thus, they might find some cognitive resources available to solve their problem. Successful arbitration techniques sometimes involve taking the other person's point of view and arguing for it. So if Chloe is asked to argue in favour of, say, desert and Daniel is directed to focus on the importance of friendship, this may reduce the effect of the bias. I do not have space to explore that avenue here, but it is an avenue worth pursuing. ${ }^{17}$ If such measures are effective then even if Broome's account is not insulated from self-serving biases, at least it offers enough specificity and direction to help agents identify the areas that are most likely to be causing disputes. ${ }^{18}$

It is therefore not obvious that the self-serving bias will tend to distort the calculations that Murphy's and Broome's accounts of fairness require of moral agents to any greater extent than, for example, act-utilitarian calculations can be distorted by the self-serving bias. In contrast, the problem for Cullity appears exponentially greater than for Murphy, Broome, and forms of utilitarianism. Even though every one of these theories requires some degree of interpretation and the exercise of good judgement, each of them channels moral thinking much more narrowly than Cullity does.

\section{Conclusion}

The self-serving bias operates stealthily to prejudice judgements about what is fair, particularly in complex cases. I have argued that the self-serving bias frequently distorts judgments about what fairness requires. In particular, Cullity's account of fairness as appropriate impartiality allows so much scope for interpretation that judgements about fairness made according to Cullity's account are most likely to be adversely influenced by the self-serving bias.

For example, I have demonstrated that Cullity's limitation on the demandingness of the moral duty of beneficence is vulnerable to the self-serving bias. Without much more guidance concerning appropriate impartiality than Cullity is willing to provide, consensus about what fairness actually requires will be difficult to attain. Without an effective method of minimizing the self-serving bias in operation, an account of fairness as appropriate impartiality will be reliable only when it is clear both what form of impartiality is most appropriate and to what degree impartiality should apply. Those cases, I maintain, are scarce and unlikely to feature in disputes about fairness.

\footnotetext{
${ }^{17}$ Self-serving biases might apply to Singer's demandingness even though the demandingness is generated by a premise that is self-sacrificing, not self-serving. Singer's principle becomes demanding because his theory assesses each individual sacrifice iteratively: any time I can avert a great harm at relatively little personal cost, I ought to do so. But when can I stop? One suggestion is that I cannot stop until making a further donation to poverty alleviation would necessitate a morally comparable sacrifice. The question follows, what is morally comparable and who is to judge? Just as the self-serving bias affects judgments about what is fair, it may also affect a judgment about what counts as 'morally comparable'. I am indebted to an anonymous reviewer for raising this point.

${ }^{18}$ Some multinational organisations work in conjunction with psychologists to help employees recognise and reverse implicit biases. Progress in mitigating self-serving biases would depend on awareness of the biases in general, since the very nature of the bias is that we are unlikely to recognise it in ourselves.
} 
Acknowledgments I would like to thank Brad Hooker, Philip Stratton-Lake, Laura Gow, Nat Hansen, Alex Gregory and Richard Rowland for reading and discussing earlier versions of this paper. This paper has also benefitted from questions and comments from audiences at the University of Reading, the University of Salzburg and the University of South Wales. I am very grateful to two anonymous referees for Ethical Theory and Moral Practice, for their comments and suggestions.

Open Access This article is distributed under the terms of the Creative Commons Attribution 4.0 International License (http://creativecommons.org/licenses/by/4.0/), which permits unrestricted use, distribution, and reproduction in any medium, provided you give appropriate credit to the original author(s) and the source, provide a link to the Creative Commons license, and indicate if changes were made.

\section{References}

Babcock L, Loewenstein G (1997) Explaining bargaining impasse: the role of self-serving biases. J Econ Perspect 11(1):109-126

Braybrooke D (1987) Meeting needs, Princeton. Princeton University Press

Broome, J. (1990/91). Fairness. Proceedings of the Aristotelian Society, New Series, Vol. 91, p. 99.

Broome J (1998) Review: kamm on fairness. Philos Phenomenol Res 58(4):955-961

Cullity G (2004) The moral demands of affluence. Oxford University Press, Oxford

Cullity G (2008) Public goods and fairness. Australas J Philos 86(1):1-21

Kriss P, Loewenstein G, Wang X, Weber R (2011) Behind the veil of ignorance: self-serving bias in climate change negotiations. Judgment and Decision Making 6(7):602-615

Miller D (1999) Principles of social justice. Harvard University Press, Harvard

Murphy L (2000) Moral demands in nonideal theory. Oxford University Press, Oxford

Shu L, Bazerman MH (2012) Cognitive barriers to environmental action: problems and solutions. In: Bansal P, Hoffman A (eds) The Oxford handbook of business and the natural environment. Oxford University Press, Oxford

Singer P (1972) Famine, affluence, and morality. Philosophy and Public Affairs 1:229-243

Thomson G (2005) Fundamental needs. In: Reader S (ed) The philosophy of need. Cambridge University Press, Cambridge

Wiggins D (1998) Needs, values, truth: essays in the philosophy of value, 3rd edn. Oxford, Oxford University Press 